\title{
Using granger-geweke causality model to evaluate the effective connectivity of primary motor cortex, supplementary motor area and cerebellum
}

\author{
Le Zhang $^{1 *}$, Guangjin Zhong ${ }^{1}$, Yukun Wu ${ }^{2}$, Mark G. Vangel ${ }^{3}$, Beini Jiang ${ }^{1}$, Jian Kong ${ }^{3,4^{*}}$ \\ ${ }^{1}$ Department of Mathematical Sciences of Michigan Tech University, Houghton, USA; \\ ${ }^{2}$ Center for Vaccine Development, University of Maryland Schhool of Medicine, West Baltimore Street, Baltimore, USA; \\ ${ }^{3}$ Harvard-MIT (HST) Athinoula A. Martinos Center for Biomedical Imaging, Charlestown, USA; \\ ${ }^{4}$ Department of Psychiatry, Massachusetts General Hospital, Charlestown, USA. \\ Email: zhangle@mtu.edu; kongj@,nmr.mgh.harvard.edu
}

Received 3 June 2010; revised 20 June 2010; accepted 24 June 2010.

\begin{abstract}
Currently, Granger-Geweke causality models have been widely applied to investigate the dynamic direction relationships among brain regions. In a previous study, we have found that the right hand finger-tapping task can produce relatively reliable brain response. As an extension of our previous study, we developed an algorithm based on the classical GrangerGeweke causality model to further investigate the effective connectivity of three brain regions (left primary motor cortex (M1), supplementary motor area (SMA) and right cerebellum) that showed the most robust brain activations. Our computational results not only confirm the strong linear feedback among SMA, M1 and right cerebellum, but also demonstrate that M1 is the hub of these three regions indicated by the anatomy research. Moreover, the model predicts the high intermediate node density existing in the area between SMA and M1, which will stimulate the imaging experimentalists to carry out new experiments to validate this postulation.
\end{abstract}

Keywords: Granger-Geweke Causality Model; Time Series; Computational Neuroscience; fMRI; Finger-tapping; Hand Movement; Math Modeling

\section{INTRODUCTION}

Recently, effective connectivity methods have been widely applied on the functional Magnetic Resonance Imaging $(f M R I)$ data set to investigate the dynamic directional relationships among brain regions [1-5]. In particular, in generating the testable hypothesis regarding the function of human brain networks, directional information obtained from Granger-Geweke causality model [6-
12] has played a pivotal role. The Granger-Geweke causality model[ $[7,13,14]$, which is a well-developed statistical measure based on the concept of time series forecasting [5,6,11,15-18], has been proposed for multivariate time series analysis to investigate the linear causal relationships among a set of univariate time series variables. For instance, Lin et al.[11] and Chen et al. [6] employed Granger-Geweke Causality model to investigate the interictal spike propagation and the effective connectivity of supplementary motor areas, respectively.

In a previous $f M R I$ study, we [19] investigated the test-retest reliability of electroacupuncture stimulation, a mode of sensory stimulation and finger-tapping task. We found that compared with electroacupuncture stimulation, finger-tapping task can generate significant and reliable $f M R I$ signal increases across different experimental sessions. Thus, in this study, we propose to reanalyze the finger-tapping data set (six subjects each repeated in 6 identical experimental sessions) using Granger-Geweke causality model to elucidate the effective connectivity among the key regions involved in the finger tapping. These three regions are left primary motor area $(M 1)$, medial supplementary motor area (SMA) and right cerebellum. Several reasons motivated selection of the data sets. First, right hand finger tapping task can produce robust and reliable $f M R I$ signal increase; secondly, the brain regions involved in finger-tapping and their interaction are relatively clear.

The $f M R I$ technology provides different types of time series for brain research, either stationary or non-stationary time series, but the classical Granger-Geweke Causality model can only process the stationary time series. For this reason, the aim of this research is developing a general algorithm developed from the Granger-Geweke Causality model to analyze the various types of $f M R I$ 
time series, such as our previous experimental data [19]. This algorithm is briefly described as follows. First, since $f M R I$ will provide us a stationary or nonstationary time series, the augmented Dickey-Fuller $(A D F)$ unit root test [20-22] will be implemented to test the stationarity of raw data. If the data are nonstationary, the plot of autocorrelation function will be applied to check the patterns and choose an appropriate smoothing technique to transform the raw data to stationary data. Next, the approximation to the critical values of Schwarz's Bayesian information criterion (SBIC) is computed by ARFIT algorithm [23] to determine the order of autoregressive equation of the Granger-Geweke Causality model. Consequently, a time series autoregressive model with appropriate order will be developed to fit smoothed fMRI data. Lastly, the confidence intervals will be constructed for the measures of feedback. In the study, the results of the model not only agree with our previous experimental findings [19] that there are strong correlations among $S M A, M 1$ and cerebellum, but also match the observations of the anatomy [24] that both SMA and cerebellum project to $M 1$.

\section{MATERIALS AND METHODS}

\subsection{Experimental Material and Methods}

In the present study, we reanalyzed the data from a previous study (experimental details described in the original paper). In summary, 6 healthy right hand subjects were included in this study. All experiments were conducted with the written consent of each subject and approved by the Massachusetts General Hospital's Institutional Review Board.

\subsection{Experimental Procedures}

Each subject participated in 6 identical $f M R I$ scanning sessions. Sessions 1 and 2 were separated by 20-30 minutes. Sessions 2 and 3 were separated by 3-6 days. After Session 3 , the interval between subsequent sessions was 7-21 days. The block design was applied. The fMRI scan started with 30s baseline, four 30s blocks of stimulation (ON, right finger-tapping), were separated by four rest periods (OFF) of 30s, 60s, 30s and 30s respectively (please see Figure 1(a) in our previous paper [19] for more detailed experimental paradigm).

\section{3. fMRI Data Acquisition and Analysis}

All brain imaging was performed with a 3-axis gradient head coil in a 3 Tesla Siemens MRI System (Erlagen, Germany) equipped for echo planar imaging. After automated scout and shimming procedures, functional $M R$ images were acquired using gradient echo $T 2 *$-weighted sequence with $T R 2000 \mathrm{~ms}, T E 40 \mathrm{msec}$ and a flip angle of 90 degrees. Thirty slices ( $4 \mathrm{~mm}$ thick, $1 \mathrm{~mm}$ skip) ori- ented parallel to the $A C-P C$ plane were collected to provide whole brain coverage. A high resolution $3 D M P$ $R A G E$ sequence was also collected. Pre-processing and statistical analysis were performed using SPM2 software (Wellcome Department of Cognitive Neurology). Preprocessing began with motion correction. All functional runs were realigned to the first volume acquired in the scan session. We set a movement threshold of $2 \mathrm{~mm}$ within a scan to eliminate subjects with excessive head movement. However, none of the subjects had head movements that exceeded this threshold. Thus, all data were used for this analysis. All functional runs were normalized to $M N I$ stereotactic space and spatially smoothed with an $8 \mathrm{~mm}$ Gaussian kernel. A separate general linear model $(G L M)$ for each session was specified across each subject with regressors for the difference from baseline [25]. Global signal scaling was applied. Low-frequency noise was removed with a high-pass filter applied with default values (120 second) to the fMRI time series at each voxel. For each individual session, a threshold of $\mathrm{p}$ $<0.005$ uncorrected with 10 contiguous voxels was used for finger-tapping; then for each predefined $R O I$, left $M I$, $S M A$ and right cerebellum, we performed Granger causality analysis on the average time courses of voxels in 3 distinct regions, with each region defined by extracting a sphere of radius $3 \mathrm{~mm}$ around the peak activation voxel.

\section{MATHEMATICAL MODEL}

\subsection{Granger-Geweke Causality Model}

In this study, the Granger-Geweke Causality model is employed as the major tool to analyze the $f M R I$ imaging data and to reveal the relationships among those brain regions of interest. Consider two zero-mean vector timeseries $X$ and $Y$. The time-indexed observations are denoted as $x_{t}$ and $y_{t}$, where $t=1, \ldots, n$ is the time index. $X, Y$ can be modeled as autoregressive $(A R)$ processes of order $p$ as

$$
\begin{aligned}
& x_{t}=\sum_{i=1}^{p} a_{1 i} x_{t-i}+u_{1 t}, \\
& y_{t}=\sum_{i=1}^{p} b_{1 i} y_{t-i}+v_{1 t}, \\
& x_{t}=\sum_{i=1}^{p} a_{2 i} x_{t-i}+\sum_{i=1}^{p} c_{2 i} y_{t-i}+u_{2 t}, \\
& y_{t}=\sum_{i=1}^{p} b_{2 i} x_{t-i}+\sum_{i=1}^{p} d_{2 i} y_{t-i}+v_{2 t},
\end{aligned}
$$

where $a_{1 i}, \mathrm{~b}_{1 i}, a_{2 i}, b_{2 i}, c_{2 i}, d_{2 i}$ are coefficients of $A R$ models, and $u_{1 t}, v_{1 t}, u_{2 t}, v_{2 t}$ are the zero-mean residuals. Their variances are $\Sigma_{1}, T_{1}, \Sigma_{2}, T_{2}$, respectively. Let $U_{1 n \times k}, V_{1 n \times l}, U_{2 n \times k}, V_{2 n \times l}$ be the re- 
spective residual matrix of Eq.1 through 4, the variances can be estimated by ordinary least squares (OLS) method, such that $\Sigma_{1}=U_{1}^{T} U_{1} / n, T_{1}=V_{1}^{T} V_{1} / n \quad \Sigma_{2}=$ $U_{2}^{T} U_{2} / n, \mathrm{~T}_{2}=V_{2}^{T} V_{2} / n$. Then the measure of linear feedback is computed by Eq.5 and 6.

$$
\begin{gathered}
F_{Y \rightarrow X}=\ln \left(\left|\Sigma_{1}\right| /\left|\Sigma_{2}\right|\right), \\
F_{X \rightarrow Y}=\ln \left(\left|\mathrm{T}_{1}\right| /\left|\mathrm{T}_{2}\right|\right),
\end{gathered}
$$

where $F_{Y \rightarrow X}$ indicates the strength of time series $Y$ Granger-causing $X$, and $F_{X \rightarrow Y}$ indicates the strength of time series $X$ Granger-causing $Y$. The measure of instantaneous linear feedback is computed by Eq.7.

$$
F_{X \cdot Y}=\ln \left(\left|T_{2}\right| \cdot\left|\Sigma_{2}\right| /|\Gamma|\right)
$$

where $\Gamma$ in Eq.8 is the covariance matrix

$$
\Gamma=\operatorname{var}\left(\begin{array}{l}
u_{2 t} \\
v_{2 t}
\end{array}\right)=\left[\begin{array}{ll}
\Sigma_{2} & C \\
C^{\prime} & \mathrm{T}_{2}
\end{array}\right]
$$

and $\mathrm{C}$ denotes the covariance of $u_{2 t}, v_{2 t}$. The measure of linear dependence is the sum of the measures of the three types of linear feedback, which is referred as $F_{X, Y}$ in Eq.9:

$$
F_{X, Y}=F_{X \rightarrow Y}+F_{Y \rightarrow X}+F_{X \cdot Y}
$$

Typically, a time series can be described as either stationary or non-stationary, depending on the constancy of its statistical properties [26-28]. The stationary time series should have constant mean and variance over time as well as covariance which is a function of time difference only. The non-stationary time series may have either non-constant means, or non-constant variance or both, which results in spurious regression; [29, 30]. This poses a very serious problem for the estimation, and over- rejects hypothesis with $T$ (true) or $F$ (false) test statistics. Since Granger-Geweke Causality model focuses on the stationary purely nondeterministic multiple time series, the raw $f M R I$ imaging data is confirmed to be stationary. If non-stationary, differencing method is employed to transform the non-stationary data to stationary data. For this reason, a general procedure to employ GrangerGeweke Causality model is presented and described by Figure 1. Next, we will illustrate each step in detail.

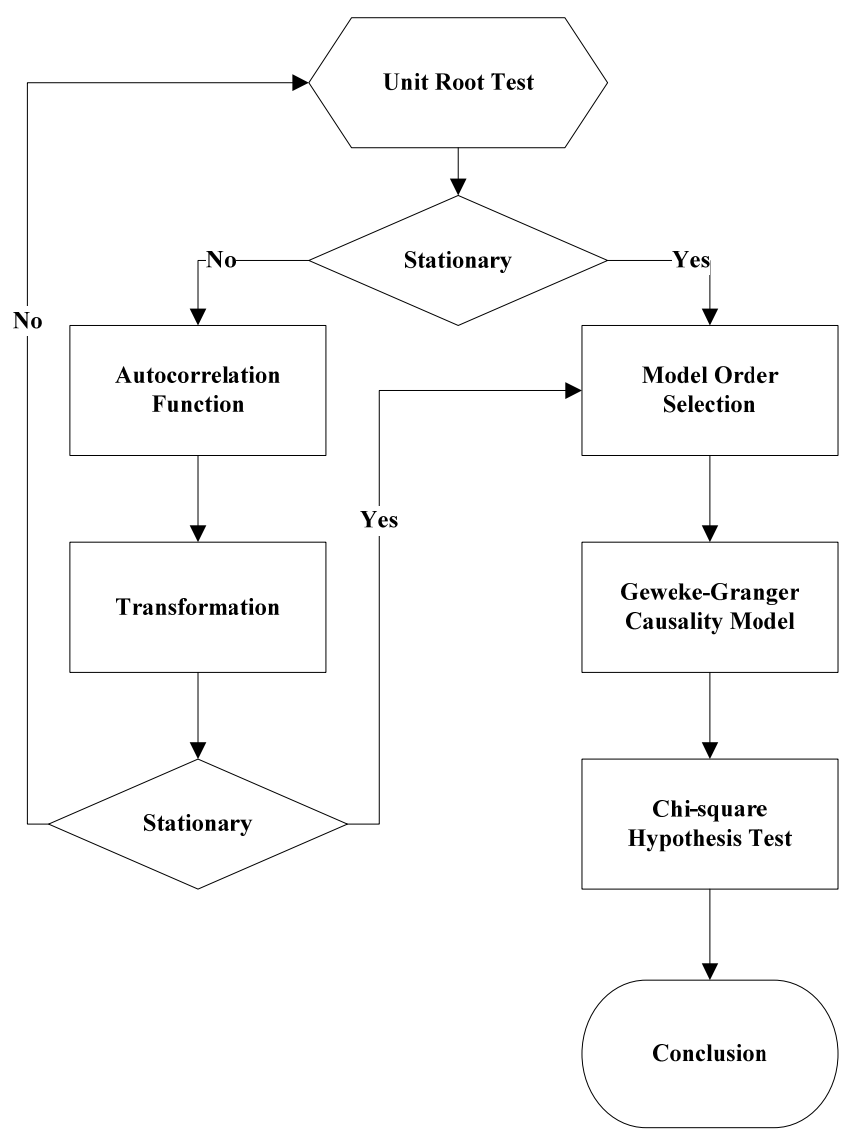

Figure 1. A general procedure to employ Granger-Geweke Causality model to investigate the relations among the interesting regions of the brain. 


\subsection{Check if the Dataset are Stationary or Non-Stationary}

The augmented Dickey-Fuller $(A D F)$ unit root test [20, 21] and the plot of autocorrelation function $(A C F)[28$, 31] are most two common methods to test whether the dataset is stationary or non-stationary.

The $A D F$ test statistics a numeric indicator such that the more negative it is, the stronger the rejection of the null hypothesis that there is a unit root (Data is not stationary) at some level of confidence. The $A D F$ test model, referred as a random walk, is described by Eq.10,

$$
x_{t}=\eta+\lambda t+\gamma x_{t-1}+\sum_{j=2}^{k} \delta_{j} x_{t-j}+\varepsilon_{t}
$$

where $k$ is the lag order, $x_{t}, x_{t-1} x_{t-i}$ are respective observations at time $t, t-1, t-j, j=2, \ldots, k$, in the time series $\mathrm{X}, \eta$ is the constant drift, $\lambda t$ is the timetrend term, $\gamma, \delta$ are coefficients, and $\varepsilon_{t}$ is the noise with mean zero and constant variance. Since the well-developed auto regression (AR) models of Granger-Geweke causality model have neither time trend nor drift processes, the current ADF test model can be simplified as Eq.11,

$$
x_{t}=\gamma x_{t-1}+\sum_{j=2}^{k} \delta_{j} x_{t-j}+\varepsilon_{t}
$$

The number of lags is determined by the sampling frequency. If the sampling frequency is large, $k$ should be small [32]. Because the time frequency of the $f M R I$ experiments is 2 seconds long, we have to set $k$ to 1 , smallest lag number in this case. And then the unit root test is carried out under the null hypothesis $\gamma=1$ against the alternative hypothesis of $\gamma<1$. Once a value for the test statistic, Eq.12 is computed, which can be compared to the relevant critical value derived in Monte Carlo study [22]

$$
D F=(\hat{\gamma}-1) / S E(\hat{\gamma})
$$

If the test statistic is smaller (this test is non symmetrical so we do not consider an absolute value) than the critical value at $\alpha$ significant level, then the null hypothesis of $\gamma=1$ is rejected and no unit root is present which means the data are stationary. Once the test results (Table 1) show that all $f M R I$ time series are non-stationary, the next step is to choose the appropriate smoothing technique by $A C F$ plot.

The $A C F$ plot is a powerful graphical tool to measure the correlation between observations at different distances apart, to check the randomness of data and to find re- peating pattern in them. Given the time series $X$ given in Eq.1, the ACF between its observations $x_{t}$ and $x_{t-i}$ is defined as

$$
\rho_{\mathrm{t}, \mathrm{t}-\mathrm{i}}=\operatorname{cov}\left(x_{t}, x_{t-i}\right) / \sigma_{x}^{2}
$$

\begin{tabular}{|c|c|c|c|c|c|c|c|c|c|}
\hline Subject & Session & M1 & SMA & cerebellum & Subject & Session & M1 & SMA & cerebellum \\
\hline \multirow{6}{*}{1} & S1 & 0.6283 & 0.6988 & 0.7338 & \multirow{6}{*}{4} & S1 & 0.6256 & 0.8848 & 0.8332 \\
\hline & S2 & 0.8834 & 0.7967 & 0.8406 & & S2 & 0.6981 & 0.7002 & 0.842 \\
\hline & S3 & 0.6915 & 0.7164 & 0.6593 & & S3 & 0.7063 & 0.7429 & 0.9074 \\
\hline & S4 & 0.6321 & 0.524 & 0.6405 & & S4 & -0.113 & -0.27 & -0.1072 \\
\hline & S5 & 0.5759 & 0.8567 & 0.8247 & & S5 & 0.8579 & 0.7953 & 0.7811 \\
\hline & S6 & 0.7728 & 0.7533 & 0.9337 & & S6 & 0.7594 & 0.9034 & 0.8619 \\
\hline \multirow{6}{*}{2} & S1 & 0.7697 & 0.6838 & 0.7179 & \multirow{6}{*}{5} & S1 & 0.7073 & 0.7633 & 0.7659 \\
\hline & S2 & 0.5857 & 0.6571 & 0.6015 & & S2 & 0.8368 & 0.8408 & 0.8512 \\
\hline & S3 & 0.7462 & 0.7352 & 0.7248 & & S3 & 0.6926 & 0.8152 & 0.8125 \\
\hline & S4 & 0.6697 & 0.7857 & 0.7402 & & S4 & 0.7143 & 0.7667 & 0.7999 \\
\hline & S5 & 0.6079 & 0.585 & 0.7134 & & S5 & 0.6698 & 0.7242 & 0.6612 \\
\hline & S6 & 0.6473 & 0.6664 & 0.8495 & & S6 & 0.8022 & 0.8728 & 0.8255 \\
\hline \multirow{6}{*}{3} & S1 & 0.7059 & 0.8223 & 0.8381 & \multirow{6}{*}{6} & S1 & 0.5849 & 0.8409 & 0.6631 \\
\hline & S2 & 0.6882 & 0.7747 & 0.7634 & & S2 & 0.807 & 0.7644 & 0.84 \\
\hline & S3 & 0.6972 & 0.8879 & 0.7782 & & S3 & 0.6915 & 0.7164 & 0.6593 \\
\hline & S4 & 0.8751 & 0.782 & 0.6341 & & S4 & 0.7688 & 0.5515 & 0.8083 \\
\hline & S5 & 0.6204 & 0.7089 & 0.6091 & & S5 & 0.6908 & 0.6905 & 0.7162 \\
\hline & S6 & 0.7709 & 0.8331 & 0.8759 & & S6 & 0.7811 & 0.8398 & 0.8479 \\
\hline
\end{tabular}

Table 1. $A D F$ test results for time series $M 1, S M A$ and cerebellum. 
where $\operatorname{cov}\left(x_{t}, x_{t-i}\right)$ is the covariance of $x_{t}$ and $x_{t-i}$, and $\sigma_{x}^{2}$ is the variance of the series [13,21]. If the autocorrelation dies out quickly in the plot (with autocorrelations on the $y$ axis and the different time lags on the $x$ axis), the series should be considered as stationary [14, 33]; especially if the autocorrelations are close to zero, the data are considered as white noise. Otherwise, the data will be considered as non-stationary time series [34].

\subsection{Data Preprocessing}

Differencing is a classical tool to transform the dataset from non-stationary to stationary. The first-order difference of a time series is the one that changes from one period to the next, that is, at time period $t$ the first order difference of series $X$ is $x_{t}-x_{t-1}$ denoted by $\Delta x_{t}$. Here, we only list the $A C F$ plots (Figure 2) for the subject 1 at Section 1 (see Table 1) restricted to the page limit. The rest of the $A C F$ plots are very similar to Figure 2. Since Figure 2 shows seasonal trends for each time series, the differencing method is adopted to remove these trends from the time series.
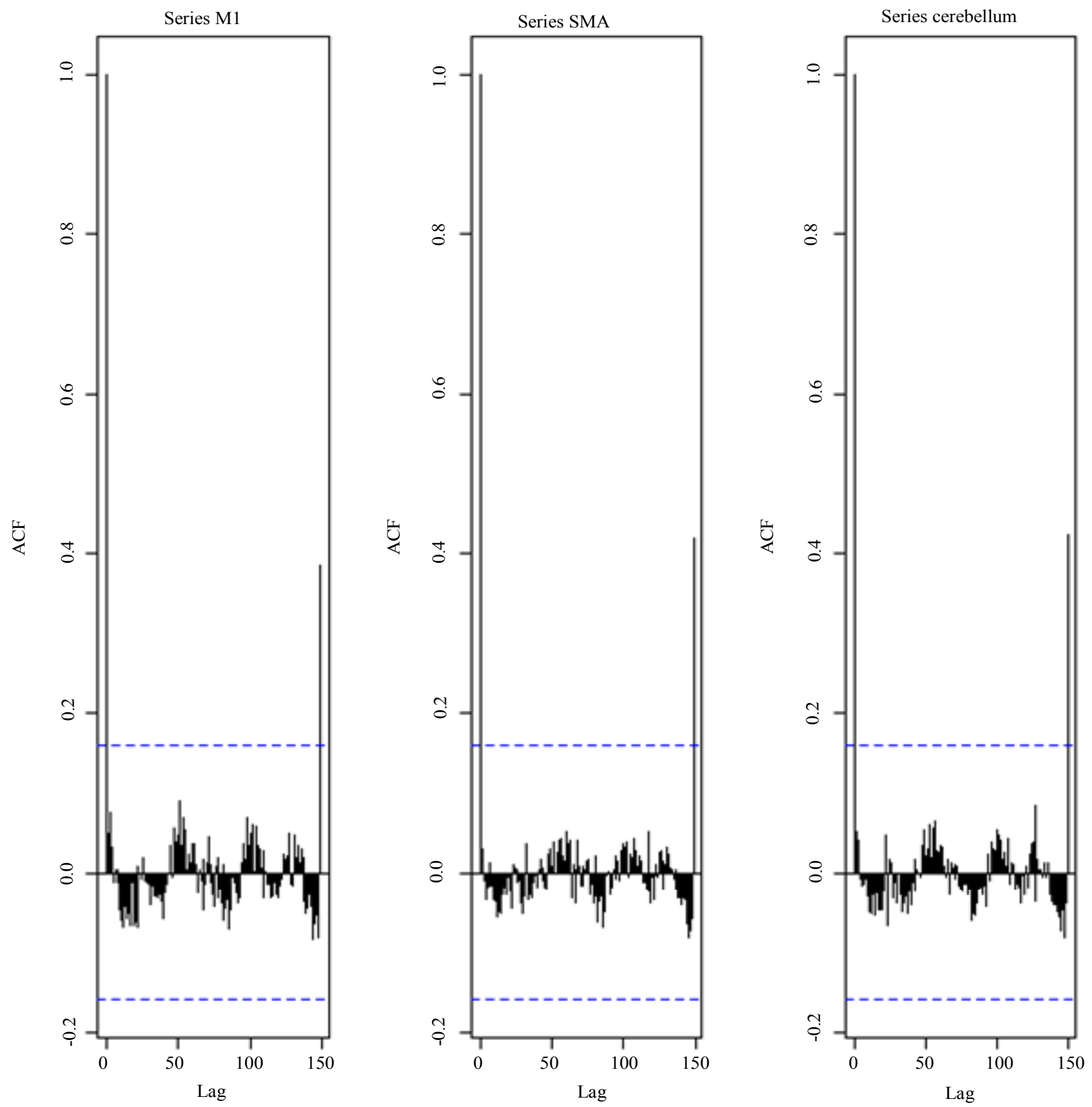

Figure 2. The $A C F$ plot of observations within each brain area for the subject 1 in Session 1 . The $x$ axis represents the number of lag; the $y$ axis represents the autocorrelation. 
Therefore, the Eq.1 through Eq. 4 can be rewritten as

$$
\begin{aligned}
& \Delta x_{t}=\sum_{i=1}^{p} a_{1 i} \Delta x_{t-i}+u_{1 t} \\
& \Delta y_{t}=\sum_{i=1}^{p} b_{1 i} \Delta y_{t-i}+v_{1 t} \\
& \Delta x_{t}=\sum_{i=1}^{p} a_{2 i} \Delta x_{t-i}+\sum_{i=1}^{p} c_{2 i} \Delta y_{t-i}+u_{2 t} \\
& \Delta y_{t}=\sum_{i=1}^{p} b_{2 i} \Delta x_{t-i}+\sum_{i=1}^{p} d_{2 i} \Delta y_{t-i}+v_{2 t}
\end{aligned}
$$

After the first-order difference, $A D F$ test is employed again of $\Delta x_{t}$ to evaluate whether the treated dataset is stationary or non-stationary. If it is still non-stationary, the second-order difference $\left(\Delta^{2} x_{t}=x_{t}-2 x_{t-1}+x_{t-2}\right)$ should be applied. However, if the series need differenting more than twice we should use other methods, such as $\log$ transformation. In the results section of the study, we are going to discuss the data preprocessing result in detail.

\subsection{Model Selection}

After the stationary data set is generated, the order of the Eq.14 to 17 by computing the approximation to the criteria values of Schwarz's Bayesian information criterion (SBIC) is identified with ARFIT algorithm [23,35]. SBIC is an information criterion used for goodness-of-fit mod- el selection for fixed effects models with different number of parameters at some significance level, and the one with lower $S B I C$ fits the data better. The SBIC is descrybed as Eq.18.

$$
S B I C=-2 \cdot \ln L+k \cdot \ln n
$$

where $\mathrm{n}$ is the number of observations, $k$ is the number of free parameters to be estimated, and $L$ is the maximized value of the likelihood function for the estimated model.

\subsection{Linear Feedback Calculation}

After the order of $A R$ models is determined, the linear feedback for each pair of brain regions by Eq.5 and 6 is computed. Then, the conventional large-sample distribution theory is used to test the null hypothesis that a given measure of feedback zero. As indicated by Geweke's research[7] if $F_{Y \rightarrow X}=0$, then $n \hat{F}_{Y \rightarrow X} \stackrel{\alpha}{\sim} \chi^{2}(k l p)$; if $F_{X \rightarrow Y}=0$, then $n \hat{F}_{Y \rightarrow X} \stackrel{\alpha}{\sim} \chi^{2}(k l p)$; if $F_{X \cdot Y}=0$, then $n \hat{F}_{X \cdot Y} \stackrel{\alpha}{\sim} \chi^{2}(k l)$; if $F_{X, Y}=0$, then $n \hat{F}_{X, Y} \stackrel{\alpha}{\sim} \chi^{2}$ $(k l(2 p+1))$. The $k, l$ are the number of columns in the residual matrix $U_{1_{n \times k}}, V_{1 n \times l}$. And $p$ is the lag of autoregressive models. With respect to this null hypothesis test, we can have the following eight relations (Eqs.19.1-19.8) between two series $X$ and $Y$ [36] described by Figure 3.

\begin{tabular}{lcrr}
\hline \multicolumn{1}{c}{ Relations } & Sign & equation \\
\hline$X$ and $Y$ are independent, if $F_{X, Y}=0$. & $(\mathrm{x}, \mathrm{y})$ & $(19.1)$ \\
\hline Instantaneous causality only without direction, if $F_{X, Y} \neq 0, F_{Y \rightarrow X}=0, F_{X \rightarrow Y}=0$ & $(\mathrm{x}-\mathrm{y})$ & $(19.2)$ \\
\hline$X$ causes $Y$, with instantaneous causality, if $F_{X, Y} \neq 0, F_{Y \rightarrow X}=0, F_{X \rightarrow Y} \neq 0$ & $(\mathrm{x} \rightarrow \mathrm{y})$ & $(19.3)$ \\
\hline$X$ causes $Y$, without instantaneous causality, if $F_{X, Y}=0, F_{Y \rightarrow X}=0, F_{X \rightarrow Y} \neq 0$ & $(\mathrm{x}=>\mathrm{y})$ & $(19.4)$ \\
\hline$Y$ causes $X$, with instantaneous causality, if $F_{X, Y} \neq 0, F_{Y \rightarrow X} \neq 0, F_{X \rightarrow Y}=0$ & $(\mathrm{x} \leftarrow \mathrm{y})$ & $(19.5)$ \\
\hline$Y$ causes $X$, without instantaneous causality, if $F_{X, Y}=0, F_{Y \rightarrow X} \neq 0, F_{X \rightarrow Y}=0$ & $(\mathrm{x}<=\mathrm{y})$ & $(19.6)$ \\
\hline Feedback with instantaneous causality, if $F_{X, Y} \neq 0, F_{Y \rightarrow X} \neq 0, F_{X \rightarrow Y} \neq 0$ & $(\mathrm{x} \leftrightarrow \mathrm{y})$ & $(19.7)$ \\
\hline Feedback without instantaneous causality $F_{X, Y}=0, F_{Y \rightarrow X} \neq 0, F_{X \rightarrow Y} \neq 0$ & $(\mathrm{x} \Leftrightarrow \mathrm{y})$ & $(19.8)$ \\
\hline
\end{tabular}

\section{RESULTS}

Stationary check for the dataset: Table 1 shows the $A D F$ test results for time series $M 1, S M A$, and cerebellum. It indicates that each time series is non-stationary at $10 \%$ significant level. Figure 2 shows the $A C F$ plots of each time series for subject 1 at session 1 and the $A C F$ plots of the rest of persons are similar with Figure 2. For this reason, we should employ differencing method to transform the dataset from non-stationary to stationary time series.

Data transformation from non-stationary to stationary time series: Table 2 shows $A D F$ test results for time series $M 1, S M A$, and cerebellum after the first-order differencing method is applied. Now each time series is stationary 


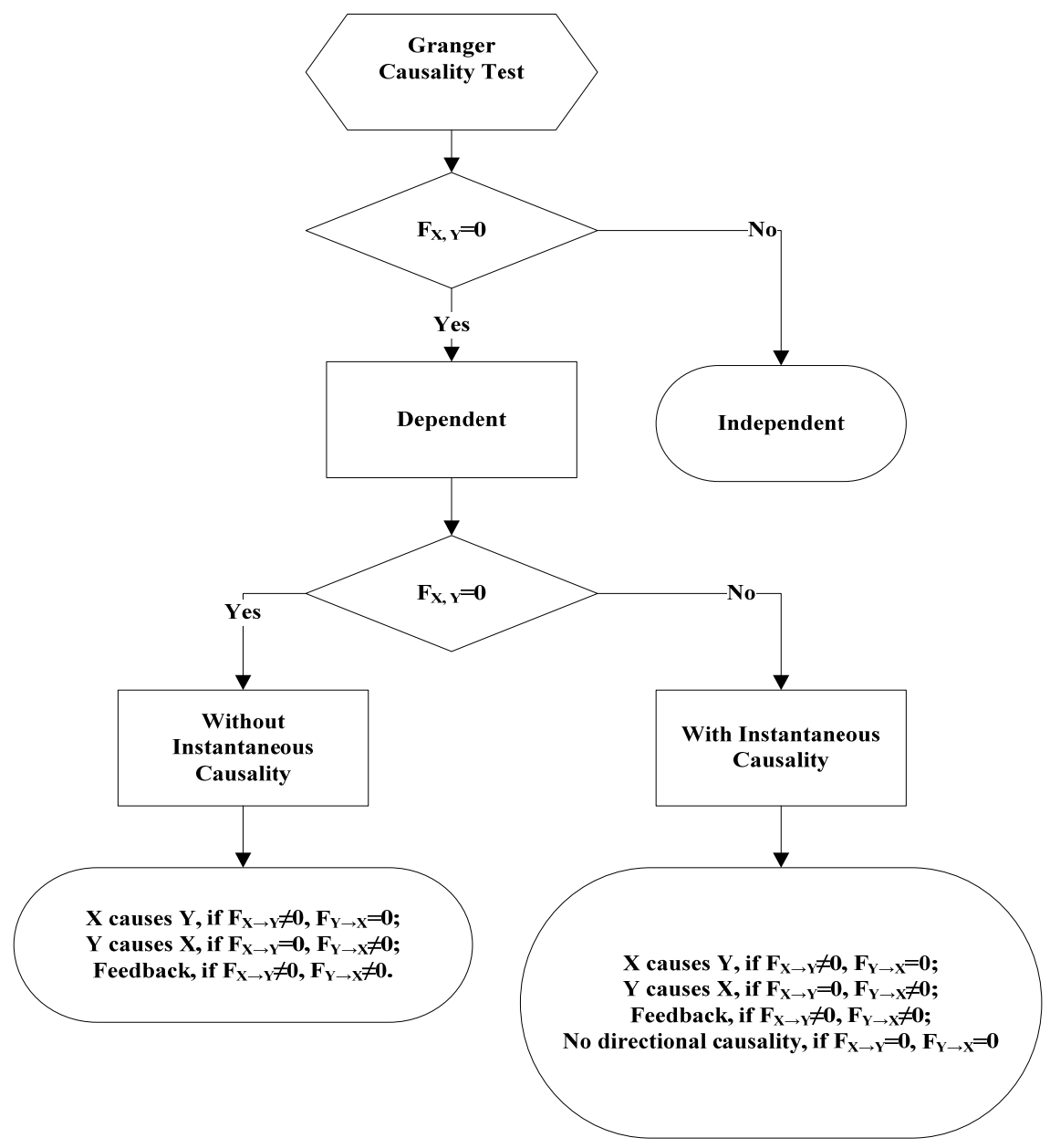

Figure 3. The relations between two time series.

Table 2. $A D F$ test results for first-order differenced time series $M 1, S M A$ and cerebellum.

\begin{tabular}{|c|c|c|c|c|c|c|c|c|c|}
\hline Subject & Session & M1 & SMA & cerebellum & Subject & Session & M1 & SMA & cerebellum \\
\hline \multirow{5}{*}{1} & $\mathrm{~S} 1$ & -6.2401 & -5.1229 & -5.0559 & \multirow{5}{*}{4} & $\mathrm{~S} 1$ & -7.0335 & -5.207 & -3.9694 \\
\hline & $\mathrm{S} 2$ & -4.3975 & -4.481 & -4.7884 & & $\mathrm{~S} 2$ & -4.8556 & -7.1719 & -3.3872 \\
\hline & $\mathrm{S} 3$ & -5.1525 & -5.4316 & -4.5684 & & $\mathrm{~S} 3$ & -5.7965 & -5.4341 & -3.1331 \\
\hline & S4 & -7.2745 & -7.9421 & -6.2819 & & S4 & -11.284 & -10.8797 & $-12,0458$ \\
\hline & S5 & -6.7513 & -3.1335 & -5.3833 & & S5 & -5.1912 & -4.3987 & -4.286 \\
\hline \multirow{6}{*}{2} & $\mathrm{~S} 1$ & -4.613 & -7.0375 & -5.0471 & \multirow{6}{*}{5} & $\mathrm{~S} 1$ & -4.8346 & -5.3248 & -4.772 \\
\hline & $\mathrm{S} 2$ & -6.8688 & -6.883 & -6.3738 & & $\mathrm{~S} 2$ & -2.8913 & -3.9684 & -2.2962 \\
\hline & $\mathrm{S} 3$ & -4.4981 & -4.0534 & -5.9015 & & S3 & -4.6129 & -4.6122 & -3.9287 \\
\hline & S4 & -4.916 & -5.6706 & -4.2594 & & $\mathrm{~S} 4$ & -4.4593 & -4.5542 & -5.1556 \\
\hline & S5 & -7.5981 & -6.8371 & -6.4166 & & S5 & -4.4734 & -6.0808 & -6.0491 \\
\hline & S6 & -7.4277 & -8.4613 & -3.4288 & & S6 & -2.9585 & -2.5292 & -3.6465 \\
\hline \multirow{5}{*}{3} & $\mathrm{~S} 2$ & -3.0548 & -4.6281 & -4.3999 & \multirow{5}{*}{6} & $\mathrm{~S} 2$ & -4.3165 & -5.5688 & -3.4785 \\
\hline & $\mathrm{S} 3$ & -4.066 & -3.7752 & -5.0195 & & $\mathrm{~S} 3$ & -5.1525 & -5.4316 & -4.5684 \\
\hline & S4 & -3.848 & -4.8816 & -6.1045 & & S4 & -4.0086 & -5.6379 & -4.9253 \\
\hline & S5 & -5.6978 & -4.8186 & -5.2083 & & S5 & -6.7513 & -3.1335 & -5.3833 \\
\hline & S6 & -2.8161 & -4.5713 & -2.4998 & & S6 & -3.2064 & -3.5299 & -3.2231 \\
\hline
\end{tabular}


and the Hochberg's [37] step-up multiple test procedure is implemented. This procedure is based on the individual P-value (Table 3) calculated by $A D F$ test and concluded that the stationarity assumption has been satisfied. Figure 4 describes the $A C F$ plot of these time series for subject 1 in session 1 and the $A C F$ plots of the rest of persons are similar with Figure 4 . Figure 4 shows that seasonal trend has been removed from time series for subject 1 in session 1 after first-order differencing. Our results show that $A C F$ plots for the remaining subjects are similar.

Order selection for the Geweke-Granger causality model: By Eq.18, we evaluated the order $p$ of the GewekeGranger models with smallest $S B I C$ values. With respect to the time interval of the previous $f M R I$ experiments (2 seconds) [19], the candidate order $p$ is limited from 1,2 and 3 . And the results in Table 4 show when $p=1$, for most of sessions, Geweke-Granger model will receive minimum $S B I C$ value. Therefore, we choose $p=1$ as the order of the model.

Table 3. The p-value of $A D F$ test.

\begin{tabular}{|c|c|c|c|c|c|c|c|c|c|}
\hline Subject & Session & M1 & SMA & cerebellum & Subject & Session & M1 & SMA & cerebellum \\
\hline \multirow{6}{*}{1} & $\mathrm{~S} 1$ & $4.88 \cdot 10^{-9}$ & $9.79 \cdot 10^{-7}$ & $1.32 \cdot 10^{-6}$ & \multirow{6}{*}{4} & $\mathrm{~S} 1$ & $8.18 \cdot 10^{-11}$ & $6.71 \cdot 10^{-7}$ & 0.000115 \\
\hline & $\mathrm{S} 2$ & $2.15 \cdot 10^{-5}$ & $1.53 \cdot 10^{-5}$ & $4.23 \cdot 10^{-6}$ & & $\mathrm{~S} 2$ & $3.17 \cdot 10^{-6}$ & $3.92 \cdot 10^{-11}$ & 0.000917 \\
\hline & $\mathrm{S} 3$ & $8.57 \cdot 10^{-7}$ & $2.40 \cdot 10^{-7}$ & $1.07 \cdot 10^{-5}$ & & S3 & $4.3 \cdot 10^{-8}$ & $2.38 \cdot 10^{-7}$ & 0.00211 \\
\hline & $\mathrm{S} 4$ & $2.26 \cdot 10^{-11}$ & $5.86 \cdot 10^{-13}$ & $3.96 \cdot 10^{-9}$ & & S4 & $<2 \cdot 10^{-16}$ & $<2 \cdot 10^{-16}$ & $<2 \cdot 10^{-16}$ \\
\hline & S5 & $<2 \cdot 10^{-16}$ & $2.07 \cdot 10^{-9}$ & $<2 \cdot 10^{-16}$ & & S5 & $7.2 \cdot 10^{-7}$ & $2.14 \cdot 10^{-5}$ & $3.37 \cdot 10^{-5}$ \\
\hline & S6 & $1.94 \cdot 10^{-5}$ & $4.45 \cdot 10^{-8}$ & 0.00266 & & S6 & $<2 \cdot 10^{-16}$ & $3.32 \cdot 10^{-15}$ & $2.61 \cdot 10^{-10}$ \\
\hline \multirow{6}{*}{2} & $\mathrm{~S} 1$ & $8.87 \cdot 10^{-6}$ & $8 \cdot 10^{-11}$ & $1.37 \cdot 10^{-6}$ & \multirow{6}{*}{5} & $\mathrm{~S} 1$ & $<2 \cdot 10^{-16}$ & $4.56 \cdot 10^{-16}$ & $<2 \cdot 10^{-16}$ \\
\hline & $\mathrm{S} 2$ & $<2 \cdot 10^{-16}$ & $<2 \cdot 10^{-16}$ & $<2 \cdot 10^{-16}$ & & $\mathrm{~S} 2$ & $9.3 \cdot 10^{-12}$ & $2.83 \cdot 10^{-15}$ & $7.28 \cdot 10^{-12}$ \\
\hline & $\mathrm{S} 3$ & $1.43 \cdot 10^{-5}$ & $8.34 \cdot 10^{-5}$ & $2.59 \cdot 10^{-8}$ & & S3 & $9.88 \cdot 10^{-16}$ & $2.09 \cdot 10^{-13}$ & $2.22 \cdot 10^{-15}$ \\
\hline & S4 & $2.44 \cdot 10^{-6}$ & $7.84 \cdot 10^{-8}$ & $3.74 \cdot 10^{-5}$ & & $\mathrm{~S} 4$ & $<2 \cdot 10^{-16}$ & $4.65 \cdot 10^{-15}$ & $<2 \cdot 10^{-16}$ \\
\hline & S5 & $3.91 \cdot 10^{-12}$ & $2.3 \cdot 10^{-10}$ & $2.01 \cdot 10^{-9}$ & & S5 & $2.29 \cdot 10^{-15}$ & $<2 \cdot 10^{-16}$ & $<2 \cdot 10^{-16}$ \\
\hline & S6 & $9.88 \cdot 10^{-12}$ & $3.17 \cdot 10^{-14}$ & 0.000797 & & S6 & $1.4 \cdot 10^{-10}$ & $2.89 \cdot 10^{-10}$ & $3.97 \cdot 10^{-13}$ \\
\hline \multirow{6}{*}{3} & $\mathrm{~S} 1$ & $4.49 \cdot 10^{-5}$ & $1.34 \cdot 10^{-5}$ & 0.0178 & \multirow{6}{*}{6} & $\mathrm{~S} 1$ & $3.06 \cdot 10^{-14}$ & $<2 \cdot 10^{-16}$ & $2.6 \cdot 10^{-16}$ \\
\hline & $\mathrm{S} 2$ & 0.0027 & $8.33 \cdot 10^{-6}$ & $2.13 \cdot 10^{-5}$ & & $\mathrm{~S} 2$ & $6.07 \cdot 10^{-11}$ & $<2 \cdot 10^{-16}$ & $3.22 \cdot 10^{-13}$ \\
\hline & $\mathrm{S} 3$ & $7.94 \cdot 10^{-5}$ & 0.000235 & $1.55 \cdot 10^{-6}$ & & $\mathrm{~S} 3$ & $<2 \cdot 10^{-16}$ & $<2 \cdot 10^{-16}$ & $<2 \cdot 10^{-16}$ \\
\hline & $\mathrm{S} 4$ & 0.00018 & $2.83 \cdot 10^{-6}$ & $9.58 \cdot 10^{-9}$ & & S4 & $1.83 \cdot 10^{-12}$ & $<2 \cdot 10^{-16}$ & $2.43 \cdot 10^{-16}$ \\
\hline & S5 & $6.89 \cdot 10^{-8}$ & $3.71 \cdot 10^{-6}$ & $6.67 \cdot 10^{-7}$ & & S5 & $1.04 \cdot 10^{-12}$ & $8.9 \cdot 10^{-12}$ & $4.93 \cdot 10^{-12}$ \\
\hline & S6 & 0.00556 & $1.06 \cdot 10^{-5}$ & 0.0136 & & S6 & $2.06 \cdot 10^{-14}$ & $1.24 \cdot 10^{-9}$ & $<2 \cdot 10^{-16}$ \\
\hline
\end{tabular}

Table 4. The order of $A R$ model for equations 14 to 17.

\begin{tabular}{|c|c|c|c|c|c|c|c|c|c|}
\hline Subject & Session & M1 & SMA & cerebellum & Subject & Session & M1 & SMA & cerebellum \\
\hline \multirow{6}{*}{1} & $\mathrm{~S} 1$ & 1 & 1 & 1 & \multirow{6}{*}{4} & S1 & 2 & 1 & 1 \\
\hline & $\mathrm{S} 2$ & 1 & 1 & 2 & & $\mathrm{~S} 2$ & 1 & 1 & 1 \\
\hline & S3 & 1 & 1 & 1 & & $\mathrm{~S} 3$ & 1 & 1 & 1 \\
\hline & S4 & 1 & 2 & 1 & & $\mathrm{~S} 4$ & 3 & 1 & 2 \\
\hline & S5 & 1 & 1 & 1 & & S5 & 1 & 1 & 1 \\
\hline & S6 & 1 & 1 & 1 & & S6 & 1 & 1 & 1 \\
\hline \multirow{6}{*}{2} & $\mathrm{~S} 1$ & 1 & 2 & 1 & \multirow{6}{*}{5} & S1 & 1 & 1 & 1 \\
\hline & $\mathrm{S} 2$ & 1 & 1 & 1 & & $\mathrm{~S} 2$ & 1 & 1 & 1 \\
\hline & $\mathrm{S} 3$ & 1 & 1 & 1 & & $\mathrm{~S} 3$ & 1 & 1 & 1 \\
\hline & $\mathrm{S} 4$ & 1 & 1 & 1 & & $\mathrm{~S} 4$ & 1 & 1 & 1 \\
\hline & S5 & 1 & 1 & 1 & & S5 & 1 & 1 & 1 \\
\hline & S6 & 2 & 2 & 1 & & S6 & 1 & 1 & 1 \\
\hline \multirow{6}{*}{3} & $\mathrm{~S} 1$ & 1 & 1 & 1 & \multirow{6}{*}{6} & $\mathrm{~S} 1$ & 2 & 2 & 1 \\
\hline & $\mathrm{S} 2$ & 1 & 1 & 1 & & $\mathrm{~S} 2$ & 1 & 1 & 1 \\
\hline & S3 & 1 & 1 & 1 & & S3 & 1 & 1 & 1 \\
\hline & S4 & 1 & 1 & 1 & & $\mathrm{~S} 4$ & 1 & 1 & 1 \\
\hline & S5 & 1 & 1 & 1 & & S5 & 1 & 1 & 1 \\
\hline & S6 & 1 & 1 & 1 & & S6 & 1 & 1 & 1 \\
\hline
\end{tabular}



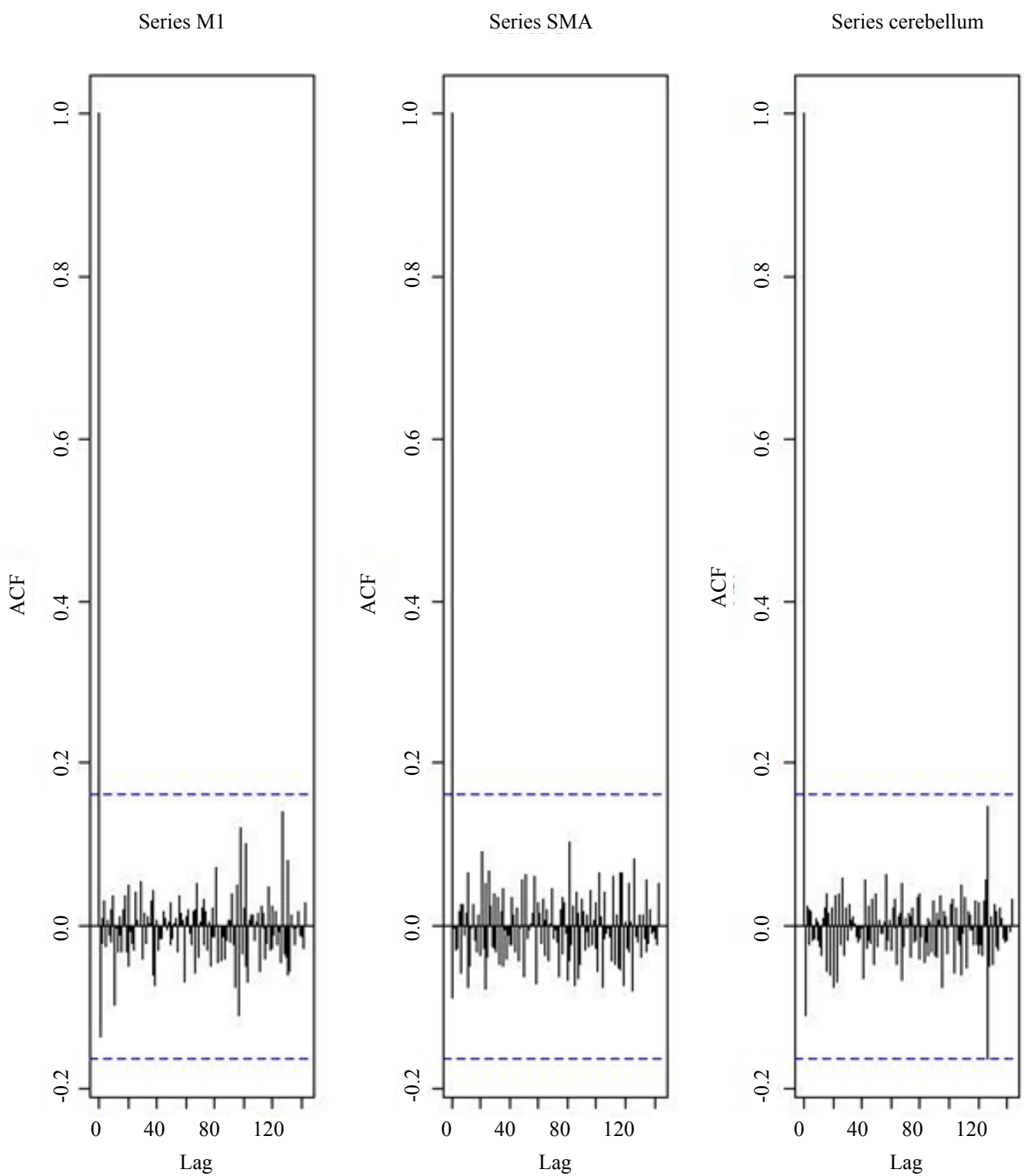

Figure 4. The $A C F$ plot of first-order differenced observations within each brain area for the subject 1 in session 1. The $x$ axis represents the number of lag; the $y$ axis represents the autocorrelation.

Causality among different brain regions: The Table 5 lists the directions among different brain regions by session. The sign is introduced by the Eqs.19.1-19.8. It reveals the following emergent phenomenon.

1) Most of the relations are instantaneous causality only without direction, $\mathrm{X}$ causes $\mathrm{Y}$ with instantaneous causality $(\mathrm{X} \rightarrow \mathrm{Y})$ and $\mathrm{Y}$ causes $\mathrm{X}$ with instantaneous causality $(\mathrm{Y} \rightarrow \mathrm{X})$. In the rest of the discussion, we denote the directed relation as $\mathrm{X}$ causes $\mathrm{Y}$ with instantaneous causality and $\mathrm{Y}$ causes $\mathrm{X}$ with instantaneous causality.

2) There should be strong directed relations between M1 and cerebellum, because we detect sixteen signals between these regions regarding to thirty six experim- ents as well as ten times the direction is from cerebellum to $M 1$ and six times from $M 1$ to cerebellum.

3) There should be strong directed relations between $M 1$ and SMA because we detect ten signals between these regions regarding to thirty six experiments as well as five times the direction is from cerebellum to $M 1$ and five times from $M 1$ to cerebellum.

Table 6 lists the directions among different brain regions by subject, which explores the following emergent phenomena.

1) It shows that all the subjects responded to the stimulation during right finger-tapping task.

2) Except one subject, the rest of the five subjects ha- 
ve similar response pattern to the stimulation.

3)As we discussed in Eq.9, the sum of the measure of linear dependence $F_{X, Y}$ is the sum of directed linear feedback $F_{X \rightarrow Y}$ and $F_{Y \rightarrow X}$ as well as the instantaneous linear feedback $\left(F_{X \cdot Y}\right)$. In our study, the instantaneous linear feedback $\left(F_{X \cdot Y}\right)$ accounts for highest percentage of the sum of the measure of linear dependence $F_{X, Y}$. In most cases, the percentage is more than $90 \%$.

\section{DISCUSSION AND CONCLUSION}

This study applied Granger-Geweke Causality model to investigate the effective connectivity of $M 1, S M A$ and cerebellum during the finger-tapping task. Eq.9 shows that linear dependence $F_{X, Y}$ has three components, i.e., instantaneous linear feedback $\left(F_{X \cdot Y}\right)$, directed linear feedback $\left(F_{X \rightarrow Y}\right.$ and $\left.F_{Y \rightarrow X}\right)$. Especially, instantaneous linear feedback $\left(F_{X \cdot Y}\right)$ describes the impact between

Table 5. The relations between multivariate brain areas investigated by $f M R I$ sort by sessions. (S1,S2, S3 S4,S5, S6 represent the number of sessions).

\begin{tabular}{|c|c|c|c|c|c|c|c|c|c|}
\hline & subject & M1,SMA & M1, cerebellum & SMA, cerebellum & & subject & M1,SMA & M1, cerebellum & SMA, cerebellum \\
\hline \multirow{6}{*}{ S1 } & 1 & - & $\rightarrow$ & - & \multirow{6}{*}{ S4 } & 1 & - & - & - \\
\hline & 2 & - & - & - & & 2 & - & - & - \\
\hline & 3 & - & $\leftarrow$ & - & & 3 & - & - & - \\
\hline & 4 & $\Leftrightarrow$ & $\leftarrow$ & $\rightarrow$ & & 4 & $\leftarrow$ & $\leftarrow$ & - \\
\hline & 5 & $\leftarrow$ & $\leftarrow$ & - & & 5 & - & $\leftarrow$ & $\leftarrow$ \\
\hline & 6 & - & - & - & & 6 & $\leftarrow$ & - & $\leftarrow$ \\
\hline \multirow{6}{*}{ S2 } & 1 & $\rightarrow$ & $\rightarrow$ & - & \multirow{6}{*}{ S5 } & 1 & - & - & - \\
\hline & 2 & - & - & - & & 2 & - & - & - \\
\hline & 3 & - & $\leftarrow$ & $\leftarrow$ & & 3 & - & - & - \\
\hline & 4 & - & - & - & & 4 & $\rightarrow$ & $\Leftrightarrow$ & - \\
\hline & 5 & - & $\leftarrow$ & - & & 5 & - & - & - \\
\hline & 6 & $\rightarrow$ & $\rightarrow$ & - & & 6 & $\leftarrow$ & $\rightarrow$ & $\rightarrow$ \\
\hline \multirow{6}{*}{ S3 } & 1 & - & - & - & \multirow{6}{*}{ S6 } & 1 & - & $\leftarrow$ & $\leftarrow$ \\
\hline & 2 & - & - & - & & 2 & - & - & - \\
\hline & 3 & $\leftarrow$ & $\rightarrow$ & $\rightarrow$ & & 3 & - & $\leftarrow$ & - \\
\hline & 4 & - & $\leftarrow$ & - & & 4 & - & - & $\leftarrow$ \\
\hline & 5 & - & - & - & & 5 & $\rightarrow$ & - & - \\
\hline & 6 & - & $\rightarrow$ & - & & 6 & $\rightarrow$ & - & - \\
\hline
\end{tabular}

Table 6. The relations between multivariate brain areas investigated by $f M R I$ sort by subjects. The number in the paragraph shows the ratio of $F_{X \cdot Y} / F_{X, Y}$.

\begin{tabular}{|c|c|c|c|c|c|c|c|c|c|}
\hline subject & & M1,SMA & M1, cerebellum & SMA, cerebellum & subject & & M1,SMA & M1, cerebellum & SMA, cerebellum \\
\hline \multirow{6}{*}{1} & S1 & - & $\rightarrow(99.5 \%)$ & - & \multirow{6}{*}{4} & S1 & $\Leftrightarrow$ & $\leftarrow(95.4 \%)$ & $\rightarrow(95.4 \%)$ \\
\hline & S2 & $\rightarrow(96.1 \%)$ & $\rightarrow(92.4 \%)$ & - & & S2 & - & - & - \\
\hline & S3 & - & - & - & & S3 & - & $\leftarrow(96.1 \%)$ & - \\
\hline & S4 & - & - & - & & S4 & $\leftarrow(67.9 \%)$ & $\leftarrow(24.5 \%)$ & - \\
\hline & S5 & - & - & - & & S5 & $\rightarrow(91.6 \%)$ & $\Leftrightarrow$ & - \\
\hline & S6 & - & $\leftarrow(96.7 \%)$ & $\leftarrow(97.7 \%)$ & & S6 & - & - & $\leftarrow(93.3 \%)$ \\
\hline \multirow{6}{*}{2} & S1 & - & - & - & \multirow{6}{*}{5} & S1 & $\leftarrow(96.6 \%)$ & $\leftarrow(99.7 \%)$ & - \\
\hline & S2 & - & - & - & & S2 & - & $\leftarrow(97.7 \%)$ & - \\
\hline & S3 & - & - & - & & S3 & - & - & - \\
\hline & S4 & - & - & - & & S4 & - & $\leftarrow(96.3 \%)$ & $\leftarrow(95.4 \%)$ \\
\hline & S5 & - & - & - & & S5 & - & - & - \\
\hline & S6 & - & - & - & & S6 & $\rightarrow(95.8 \%)$ & - & - \\
\hline \multirow{6}{*}{3} & S1 & - & $\leftarrow(96.9 \%)$ & - & \multirow{6}{*}{6} & S1 & - & - & - \\
\hline & S2 & - & $\leftarrow(93.5 \%)$ & $\leftarrow(94.6 \%)$ & & S2 & $\rightarrow(96.9 \%)$ & $\rightarrow(97.5 \%)$ & - \\
\hline & S3 & $\leftarrow(94.1 \%)$ & $\rightarrow(96.7 \%)$ & $\rightarrow(96.7 \%)$ & & S3 & - & $\rightarrow(97.2 \%)$ & - \\
\hline & S4 & - & - & - & & S4 & $\leftarrow(99.2 \%)$ & - & $\leftarrow(96.5 \%)$ \\
\hline & S5 & - & - & - & & S5 & $\leftarrow(92.3 \%)$ & $\rightarrow(92.9 \%)$ & $\rightarrow(92.2 \%)$ \\
\hline & S6 & - & $\leftarrow(95.7 \%)$ & - & & S6 & $\rightarrow(96.9 \%)$ & - & - \\
\hline
\end{tabular}


time series $X$ and $Y$ at current time point. Directed linear feedback $\left(F_{X \rightarrow Y}\right.$ or $\left.F_{Y \rightarrow X}\right)$ describes how the effect of time series $X$ or $Y$ in previous $t-1$ time steps affects time series $Y$ or $X$ at time point $t$. Actually, the relation between two time series could include more than one type of linear feedback simultaneously. Therefore, Kirchgässner and Wolters [36] classified these linear feedbacks to eight relations (Eqs.19.1-19.8), which are not independent of each other.

The current results show (Table 5) that most of the pairwise relations between the brain regions are instantaneous causality only without direction (Eq.19.2). $X$ causes $Y$ with instantaneous causality (Eq.19.3) and $Y$ causes $X$ with instantaneous causality (Eq.19.5). The rest relation such as feedback without instantaneous causality (Eq.19.8) only appears twice (Table 5). We denote the relations like $X$ causing $Y$ with instantaneous causality (Eq.19.3) and $Y$ causing $X$ with instantaneous causality (Eq.19.5) as the directed relation in the rest of the discussion. Table 6 shows instantaneous causality only without direction (Eq.19.2) is the most favorite relation in the results. More importantly, the most popular relation, instantaneous linear feedback $\left(F_{X \cdot Y}\right)$ component, takes highest percentage (mostly more than $90 \%$ (Table 6)) of the sum of the linear dependency $\left(F_{X, Y}\right)$. The phenomena imply that neurology response time period should be shorter than the $f M R I$ time interval ( 2 seconds). Next, the directed relations among these brain regions were investigated. Figure 5 describes the directed relations between each pair of the brain regions. It shows strong directed relations between $M 1$ and cerebellum, $M 1$ and SMA, because there are sixteen and ten directed signals transductions occurred between the pair of these regions, respectively. Additionally, if we consider each region as a node of the brain network, Figure 6 demonstrates that $M 1$ should be a busy node, since it is involved 26 directed signal transductions. Particularly, this finding matches the anatomical observations [24] that the both SMA and Cerebellum project to $M 1$. Furthermore, Figure 5

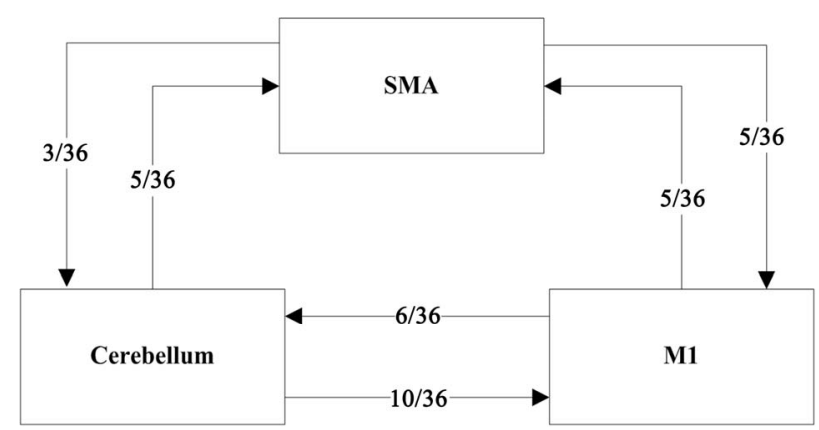

Figure 5. The directed relation between every two brain areas. The arrow indicates the direction of causality. The label of each link indicates the number of the directed signals.

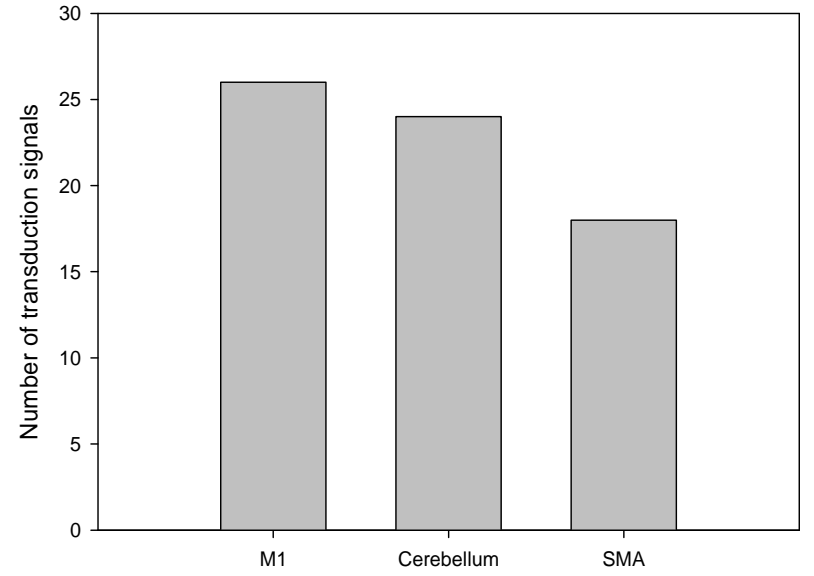

Figure 6. The number of directed signal transductions for each brain regions, $M 1$, cerebellum and SMA.

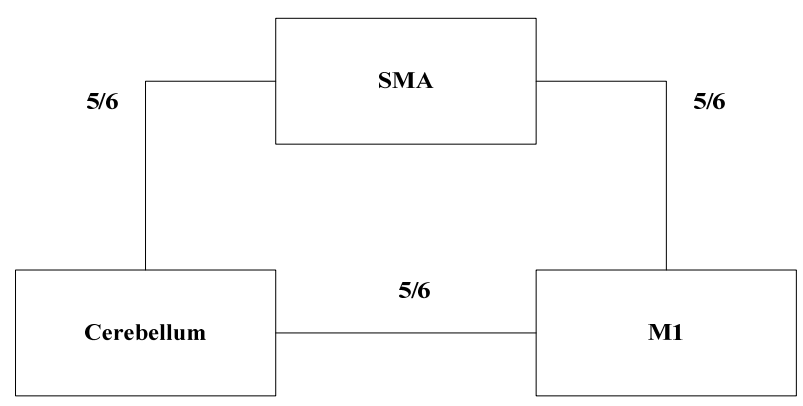

Figure 7. The directed relation between every two brain areas. The label of each link indicates the numbers of subject out of six have the relation between these two regions.

shows that SMA and cerebellum are the regions which have less directed signal transductions. Due to that, we consider the number of intermediate nodes between $S M A$ and cerebellum should be less than others which will not cause many latency of the signal transduction between these regions. Figure 7 shows the directed simulation response is stable and believable, since five out of six subjects showed the directed relations.

In summary, the results demonstrate strong linear feedback among SMA, M1 and cerebellum as our previous study. Especially, the instantaneous linear feedback plays the very important role. Also, strong directed relations were found between $M 1$ and cerebellum, $M 1$ and SMA. It derives that M1 should be the hub of these three regions and such findings agree with the study of anatomy that both $S M A$ and Cerebellum project to $M 1$. Also, as indicated in the anatomy field [24], the distance between $S M A$ and $M 1$ is the shortest, but a number of directed signals were detected (Figure 5), which implies a high intermediate node density existing in the area between these two regions. On the other hand, the distance between $S M A$ and cerebellum is much longer than $S M A$ and $M 1$, 
but very few directed relations between them can be obtained. We postulate that there are not many intermediate nodes in the area between SMA and cerebellum compared to the area between $S M A$ and $M 1$.

\section{ACKNOWLEDGEMENTS}

Funding and support for this study came from the startup funding of Michigan Tech University, NIH (NCCAM) K01 AT003883 and R21AT004497 to Jian Kong. M01-RR-01066 for Mallinckrodt General Clinical Research Center Biomedical Imaging Core, P41RR14075 for Center for Functional Neuroimaging Technologies from NCRR, and the MIND Institute. Also, we appreciate the suggestions and help from Dr. Tom Drummer.

\section{REFERENCES}

[1] Astolfi, L., Cincotti, F., Mattia, D., Salinari, S., Babiloni, C. and Basilisco, A., et al. (2004) Estimation of the effective and functional human cortical connectivity with structural equation modeling and directed transfer function applied to high-resolution EEG. Magn Reson Imaging, 22(10), 1457-1470.

[2] Brovelli, A., Ding, M.Z., Ledberg, A., Chen, Y.H., Nakamura, R. and Bressler, S.L. (2004) Beta oscillations in a large- scale sensorimotor cortical network: Directional influences revealed by Granger causality. Proceedings of the National Academy of Sciences of the United States of America, 101(26), 9849-9854.

[3] Eichler, M. (2005) A graphical approach for evaluating effective connectivity in neural systems. Philos Trans $R$ Soc Lond B Biol Sci, 360(1457), 953-967.

[4] Sato, J.R., Amaro, E.D. Takahashi, Y., Felix, M.D., Brammer, M.J. and Morettin, P.A. (2006) A method to produce evolving functional connectivity maps during the course of an fMRI experiment using wavelet-based time-varying Granger causality. Neuroimage, 31(1), 187 196.

[5] Smith, J.F., Pillai, A., Chen, K. and Horwitz, B. (2009) Identification and validation of effective connectivity networks in functional magnetic resonance imaging using switching linear dynamic systems. Neuroimage, 52(3), 1027-1040.

[6] Chen, H.F., Yang, Q., Liao, W., Gong, Q. Y. and Shen, S. (2009) Evaluation of the effective connectivity of supplementary motor areas during motor imagery using Granger causality mapping. Neuroimage, 47(4), 1844-1853.

[7] Geweke, J. (1982) The measurement of Linear dependence and feedback between multiple Time-Series rejoinder. Journal of the American Statistical Association, 77 (378), 323-324.

[8] Gow, Jr, D.W., Segawa, J.A., Ahlfors, S.P. and Lin, F.H. (2008) Lexical influences on speech perception: A Granger causality analysis of MEG and EEG source estimates. Neuroimage, 43(3), 614-623.

[9] Liao, W., Mantini, D., Zhang, Z., Pan, Z., Ding J. and Gong, Q. et al. (2010) Evaluating the effective connectivity of resting state networks using conditional Granger causality. Biological. Cybernetics, 102(1), 57-69.

[10] Liao, W., Marinazzo, D., Pan, Z., Gong, Q. and Chen, H.
Kernel Granger causality mapping effective connectivity on FMRI data. IEEE Trans Med Imaging, 28(11), 18251835.

[11] Lin, F.H., Hara, K., Solo, V., Vangel, M., Belliveau, J.W. and Stufflebeam, S.M., et al. (2009) Dynamic GrangerGeweke Causality Modeling With Application to Interictal Spike Propagation. Human Brain Mapping, 30(6), 1877-1886.

[12] Marinazzo, D., Liao, W., Chen, H. and Stramaglia, S. (2010) Nonlinear connectivity by Granger causality. Neuroimage.

[13] Box, G.E.P., Jenkins, G.M. and Reinsel, G.C. (1994) Time series analysis: Forecasting and control, Prentice Hall, New Jersey.

[14] Chatfield, C. (2001) Time-series forecasting. Chapman \& Hall/CRC.

[15] Londei, A., D’Ausilio, A., Basso, D. and Belardinelli, M. O. (2006) A new method for detecting causality in fMRI data of cognitive processing. Cogn Process, 7(1), 42-52.

[16] Nedungadi, A.G., Rangarajan, G., Jain, N. and Ding, M. Z. (2009) Analyzing multiple spike trains with nonparametric granger causality. Journal of Computational Neuroscience, 27(1), 55-64.

[17] Roebroeck, A., Formisano, E. and Goebel, R. (2005) Mapping directed influence over the brain using Granger causality and fMRI. Neuroimage, 25(1), 230-242.

[18] Zhang, Y., Chen, Y., Bressler, S.L. and Ding, M. (2008) Response preparation and inhibition: The role of the cortical sensorimotor beta rhythm. Neuroscience, 156(1), 238-246.

[19] Kong, J., Gollub, R.L., Webb, J.M., Kong, J.T., Vangel, M.G. and Kwong, K. (2007) Test-retest study of fMRI signal change evoked by electroacupuncture stimulation. Neuroimage, 34(3), 1171-1181.

[20] Greene, W.H., (2003) Econometric analysis. Prentice Ha11, New Jersey.

[21] Yaffee, R.A. and McGee, M. (2000) Introduction to time series analysis and forecasting: With applications in SAS and SPSS. Academic Press,USA.

[22] Dickey, D.A. and Fuller, W.A. (1979) Distribution of the Estimators for Autoregressive Time-Series with a Unit Root. Journal of the American Statistical Association, 74(366), 427-431.

[23] Neumaier, A. and Schneider, T. (2001) Estimation of parameters and eigenmodes of multivariate autoregressive models. Acm Transactions on Mathematical Software, 27(1), 27-57.

[24] Notle, J. (1999) The Human Brain: An Introduction to its functional anatomy. Mosby Inc., Louis.

[25] Friston, K.J., Ashburner, J.T., Kiebel, S.J., Nichols, T.E. and Penny, W.D. (2007) Statistical parametric mapping. Elsevier. Academic Press, USA.

[26] Priestley, M.B. (1981) Spectral analysis and time series. Academic Press, USA.

[27] Priestley, M.B. (1988) Non-linear and non-stationary time series analysis. Academic, USA.

[28] Wei, W. (2006) Time Series Analysis. Perrson Education, inc., Chichester.

[29] Pearl, J. (2000) Causality: Models, reasoning, and inference. Cambridge University Press, UK.

[30] Brooks, C. (2008) Introductory econometrics for finance. Cambridge University Press, UK. 
[31] Baum, C.F. (2006) An introduction to modern econometrics using Stata. Stata Press, Texas.

[32] Warner, R.M. (1998) Spectral analysis of time-series data. Guilford Press, New York.

[33] Mills, T.C. (1990) Time series techniques for economists, Cambridge University Press, UK.

[34] Clements, P. and Hendry, D.F. (1999) Forecasting nonstationary economic time series. MIT Press, Cambridge.
[35] Storch, H.V. and Zwiers, F.W. (1999) Statistical analysis in climate research. Cambridge University Press, UK.

[36] Kirchgässner, G. and Wolters, J. (2007) Introduction to modern time series analysis. Springer, New York.

[37] Hochberg, Y. (1988) A Sharper Bonferroni Procedure for Multiple Tests of Significance. Biometrika, 75(4), 800802. 